platelet function testing in neonate populations are lacking mainly due to the large blood volume requirements. To address this we developed a physiologically relevant assay on vonWillebrand factor that mimics platelet behaviour in vivo at arterial shear conditions.

Methods A prospective clinical study of blood from preterms < 32 weeks $(n=21)$, terms $(n=12)$ and adults $(n=48) .200 \mu l$ of blood for each donor was labelled with a florescent dye for visualisation of platelets in real-time. The blood was perfused over parallel plate flow chambers coated with purified vonWillebrand Factor (vWF). Platelet interactions with the surface were imaged via video microscopy at high speed (500 frames at 30 frames/second). In-vivo when platelets are exposed to activated vWF they tether, roll and translocate. For the first time ever we managed to image these platelet behaviours using a custom designed platelet tracking algorithm.

Results Preterms had significantly more numbers of static platelets $(54 \pm 55$ vs. $26 \pm 4$ and $28 \pm 2$ SEM, p < 0.0001). In the preterm cohort there were increased numbers of platelets translocating on the vWF surface $(359 \pm 25$ vs. $277 \pm 25$ and 307 \pm 10 SEM, $p=0.05$ ) compared with the terms and adults. Clinical outcomes showed significantly high levels of RDS (76.19\%), CLD (38.09\%) IVH (28.57\%) and pulmonary haemorrhage (23.8\%).

Conclusions The behaviour of platelets on vWF is significantly different in preterms and could explain the increased tendency to bleeding and development of RDS and CLD in premature neonates. The tiny volume of blood needed to perform this test has major implications for its use in a clinical setting.

\section{PS-277 FULLY AUTOMATED CONTROL OF INSPIRED OXYGEN BASED ON OXYGEN SATURATION IN PRETERM LAMBS}

${ }^{1,2} \mathrm{M}$ Hütten, ${ }^{3,4} \mathrm{TG}$ Goos, ${ }^{1} \mathrm{D}$ Ophelders, ${ }^{1} \mathrm{M}$ Nikiforou, ${ }^{1} \mathrm{E}$ Kuypers, ${ }^{1} \mathrm{M}$ Willems, ${ }^{4} \mathrm{~J}$ Dankelman, ${ }^{3} \mathrm{KKM}$ Reiss, ${ }^{1} \mathrm{BW}$ Kramer. ${ }^{1}$ Department of Paediatrics, Maastricht University Medical Centre, Maastricht, The Netherlands; '2Department of Paediatrics, Neonatology, University Clinic Medical Faculty RWTH Aachen, Germany; ${ }^{3}$ Department of Paediatrics, Division of Neonatology, Erasmus Medical Centre - Sophia Children's Hospital, Rotterdam, The Netherlands; ${ }^{4}$ Department of Biomechanical Engineering, Delft University of Technology, Delft, The Netherlands

\subsection{6/archdischild-2014-307384.577}

Background Oxygen under- and overexposure has detrimental effects on preterm infants. Controlling the oxygen saturation manually within strict targets is a notoriously difficult and time consuming task which can be facilitated by automated control.

Aims To evaluate the performance of a closed loop fraction of inspired oxygen $\left(\mathrm{FiO}_{2}\right)$ controller based on oxygen saturation $\left(\mathrm{SpO}_{2}\right)$ targets when compared to dedicated manual control.

Methods Prematurely delivered lambs (128-132 days of gestation, term $150 \mathrm{~d})$ were intubated and ventilated with volume guaranteed ventilation (Fabian HFO, Acutronic $®$ ) adjusted to arterial $\mathrm{pCO}_{2} . \mathrm{SpO}_{2}$ was measured via pulse oximetry (Radical 7, Masimo $\left.{ }^{\circledR}\right)$. After a stabilisation period of $30 \mathrm{~min}$, lambs were randomised to three hours of ventilation with automated or manual $\mathrm{FiO}_{2}$ control (caretaker-lamb ratio 1:1). Saturation targets were $90-95 \%$ according to European guidelines. Data is represented as median [IQR].

Results Twenty-two lambs were included, 11 in each group. Time spend within the target range was significantly better when the automated controller was used $(93.2 \%$ [80.6-98.9\%] vs. $84.0 \%$ [63.8-89.4\%], $\mathrm{p}_{2}$ adjustments per hour was higher when the automated controller was used (13.0 [3.0-16.4] vs. 5.7 [2.39.8], n.s.).

Conclusions Automated $\mathrm{FiO}_{2}$ control was effective in this model. These results are especially promising as we compared our device to animals receiving 1:1 care in the manual control group. In a clinical setting, the combination of automated control and dedicated caretakers might even be more effective.

\section{PS-278 AUTOMATED VERSUS MANUAL FIO2 CONTROL AT DIFFERENT SATURATION TARGETS IN PRETERM INFANTS}

${ }^{1} \mathrm{~A}$ Van Kaam, ${ }^{2} \underline{\mathrm{H} \text { Hummler, }}{ }^{3} \mathrm{M}$ Wilinska, ${ }^{4} \mathrm{~J}$ Swietlinski, ${ }^{5} \mathrm{M}$ Lal, ${ }^{6} \mathrm{~A}$ Te Pas, ${ }^{7} \mathrm{G}$ Lista, ${ }^{8} \mathrm{~S}$ Gupta, ${ }^{9} \mathrm{C}$ Fajardo, ${ }^{1} \mathrm{~W}$ Onland, ${ }^{2} \mathrm{M}$ Waitz, ${ }^{3} \mathrm{M}$ Warakomska, ${ }^{7} \mathrm{~F}$ Cavigioli, ${ }^{10} \mathrm{E}$ Bancalari, ${ }^{10} \mathrm{~N}$ Claure, ${ }^{11} \mathrm{~T}$ Bachman. ${ }^{1}$ Neonatology, Emma Children's Hospital/Academic Medical Center, Amsterdam, Netherlands; ${ }^{2}$ Neonatology, University Medical Center, Ulm, Germany; ${ }^{3}$ Neonatology, Center Medical Post Graduate Education, Warsaw, Poland; ${ }^{4}$ Neonatology, City Hospital, Ruda Slaska, Poland; ${ }^{5}$ Neonatology, James Cook University Hospital, Middlesbrough, UK; ${ }^{6}$ Neonatology, Leiden University Medical Center, Leiden, Netherlands; ${ }^{7}$ Neonatology, Vittore Buzzi Children's Hospital, Milano, Italy; ${ }^{8}$ Neonatology, University Hospital North Tees, Stockton-Cleveland, UK; ${ }^{9}$ Neonatology, Alberta Children's Hospital, Calgary, Canada; ${ }^{10}$ Neonatology, University of Miami, Miami, USA; ${ }^{11}$ Economedtrx, Lake Arrowhead, USA

\subsection{6/archdischild-2014-307384.578}

Background Preterm infants spend only 50\% of time within the target oxygen saturation $\left(\mathrm{SpO}_{2}\right)$ during manual $\mathrm{FiO}_{2}$ control $\left(\mathrm{M}-\mathrm{FiO}_{2}\right)$. Automated $\mathrm{FiO}_{2}$ control $\left(\mathrm{A}-\mathrm{FiO}_{2}\right)$ improves $\mathrm{SpO}_{2}$ targeting but it is uncertain if this applies to different $\mathrm{SpO}_{2}$ target ranges and during non-invasive support (NIVS) and mechanical ventilation (MV).

Objective To compare the efficacy of $\mathrm{A}-\mathrm{FiO}_{2}$ vs $\mathrm{M}-\mathrm{FiO}_{2}$ in keeping two different $\mathrm{SpO}_{2}$ targets during NIVS or MV.

Design/methods Preterm infants on $\mathrm{FiO}_{2}>0.21$ receiving NIVS or MV were randomised to $\mathrm{SpO}_{2}$ targets $89-93 \%$ or $91-95 \%$ and underwent $\mathrm{M}-\mathrm{FiO}_{2}$ and $\mathrm{A}-\mathrm{FiO} \mathrm{O}_{2}$ for $24 \mathrm{~h}$ each, in random sequence.

Results 80 infants (GA:26 w, age:18 d) were included (NIVS = $48, \mathrm{MV}=32$ ). Time within target increased and below target decreased during $\mathrm{A}-\mathrm{FiO}_{2}$ compared with $\mathrm{M}-\mathrm{FiO}$, especially in the lower target range. There was a reduction in time and hypoxemia episodes with $\mathrm{SpO}_{2}<80 \%$ during $\mathrm{A}-\mathrm{FiO}_{2}$. Outcomes did not differ between NIVS or MV.

Conclusions Automated $\mathrm{FiO}_{2}$ control improved $\mathrm{SpO}_{2}$ targeting across different $\mathrm{SpO}_{2}$ ranges and reduced hypoxemia with less workload during both NIVS and MV.

\begin{tabular}{|c|c|c|c|c|}
\hline & \multicolumn{2}{|c|}{ Target $89-93 \%$} & \multicolumn{2}{|c|}{ Target $91-95 \%$} \\
\hline & $\mathrm{A}-\mathrm{FiO}_{2}$ & $\mathrm{M}-\mathrm{FiO}_{2}$ & $\mathrm{~A}-\mathrm{FiO}_{2}$ & $\mathrm{M}-\mathrm{FiO}_{2}$ \\
\hline \%-time in target & $62(17)$ & $54(16)^{*}$ & $62(17)$ & $58(15)^{*}$ \\
\hline$\%$-time >target & $21(13)$ & $25(10)^{*}$ & $22(13)$ & $19(8)$ \\
\hline$\%$-time $<$ target & $17(11)$ & $21(8)^{*}$ & $17(10)$ & $23(9)^{*}$ \\
\hline$\%$-time $\mathrm{SpO}_{2}>98 \%$ & $0.2(0.0-0.8)$ & $0.7(0.1-1.6)^{*}$ & $0.7(0.2-2.1)$ & $1.7(0.7-4.3)$ \\
\hline$\%$-time $\mathrm{SpO}_{2}<\mathbf{8 0} \%$ & $1.2(0.2-2.2)$ & $2.6(1.0-4.3)^{*}$ & $0.8(0.3-2.1)$ & $2.0(0.9-5.0)^{*}$ \\
\hline \multicolumn{5}{|l|}{ Episodes $<80 \%$, } \\
\hline$>1 \mathrm{~min} / 24 \mathrm{~h}$ & $4(1-12)$ & $15(5-24)^{*}$ & $4(1-11)$ & $13(3-24)^{*}$ \\
\hline Manual $\mathrm{FiO}_{2}$ & & & & \\
\hline $\begin{array}{l}\text { adjustments/24h } \\
{ }^{*} \mathrm{p}<0.05\end{array}$ & $1(0-3)$ & $102(72-173)^{*}$ & $1(0-3)$ & $109(79-156)^{*}$ \\
\hline
\end{tabular}

